\section{Atomic collisions}

\section{H. Kleinpoppen}

Atomic and Molecular Collisions. By Harrie Massey. Pp.309. (Taylor and Francis: Basingstoke, UK, 1979.) $£ 12$.

AT LAST, one can say, there is an introductory authoritative text book available on atomic and molecular collisions. The author, Sir Harrie Massey, the grand old master and pioneer in the field, has written this introductory book shortly after the completion of the monumental and comprehensive monographs on atomic collisions (edited by him and his colleagues). The introductory book is suitable both for experts and non-experts in the field and particularly for undergraduate students. The book fills a gap and will certainly be welcomed by the scientific community.

The difficulties in preparing an introduction to atomic collisions mainly result from the nature of the subject. While introductory textbooks on solid-state physics or atomic spectroscopy, for example, can be restricted to the selection of stationary physical processes (which are dealt with by stationary quantum mechanical formalism), atomic collisions require dynamical methods and approximations over a wide range of applications. Even for the simplest colliding system such as scattering between electrons and atomic hydrogen no exact theoretical solutions are available. It was not possible to include sophisticated problems of atomic collision theory in an introductory text book. The book does contain a discussion and interpretation of the collision data required to develop basic concepts of quantum mechanics such as particles and waves and their dualism, the uncertainty principle, and the quantum mechanical description of scattering cross sections. Chapters on such topics include elementary descriptions of atomic and molecular structure. Appropriate experimental technology has been explained within the main text. Only a limited selection of topics on atomic collisions could be considered in this type of book. Nevertheless, the author has covered a broad range of the large field of atomic and molecular collisions and he has succeeded in exposing the full scope of the subject. It is obvious that the selection and detailed discussion of these most interesting topics reflect the mastery and long experience of the author as one of the pioneers in the field. The order of the topics of the book follows the traditional scheme, which is mainly guided by selecting projectiles and atomic and molecular targets: elastic and inelastic electron-atom scattering (including resonance effects, capture, recombination and attachment processes), collisions between neutral atomic and molecular systems, ion-atom collisions, photoionisation of atoms and molecules, and photodetachment of negative atomic and molecular ions. The last chapter deals with atomic collisions in the Earth's atmosphere, the solar corona and interplanetary space. Seven appendices supplement information mainly on experimental technology.

Each chapter, each section and even each line of the book is excellent, and it reveals the great devotion (and love) of Sir Harrie to the field of atomic collisions. The detailed discussions on selected topics (for example, the Ramsauer-Townsend effect, the glory and rainbow structure in atomatom collisions, or the selected collision

\section{Nuclear physics}

\section{J.M. Reid}

Elements of Nuclear Physics. By W.E. Burcham. Pp.422. (Longman: Harlow, UK, 1979.) $£ 7.95$.

THE accommodation of a rapidly expanding subject is a constant problem in the construction of undergraduate course syllabuses. The problem has been particularly severe in the case of nuclear physics over the past decade. The generally accepted separation of elementary particles (or high energy physics) as a subject in its own right has not produced any long-term amelioration in the face of the constant ferment of new ideas and approaches in both the low and high energy fields. Defined by the author of the volume under review as "the understanding of the nature of matter at a level deeper than that of the electronic structure of atoms, molecules and solids but not so deep as that of the particle structure of the proton and the neutron", nuclear physics must always find a place in some form in any course claiming to span matter from the macro to the micro level. The case for the study of the subject on the grounds of its practical applications has changed dramatically from the time of its emergence from the shadow of restricted work on nuclear based weapons in 1945. It was then, optimistically, to understand the processes about to provide a solution to the world's recognised energy problems. Today the case might be to provide a background understanding of the use of nuclear techniques in order to circumscribe the risks involved in the reluctant application processes in the ionosphere) are of highest pedagogical standard. As an introduction the book represents without any doubt a break-through; it will be a challenge and precedent for future authors. This is the more important as the research field of atomic collisions is still in an explosive state: new exciting results from atomic angular correlations, from spin effects in electron-atom scattering, and from collisions with laser excited atoms will soon require new editions of introductory textbooks. Sir Harrie has paved the way (as he often did in research) for such future tasks.

H. Kleinpoppen is Director of the Institute of Atomic Physics, University of Stirling, UK.

of these techniques to socially vital problems.

However, Professor Burcham's latest textbook is not concerned with the practical applications of nuclear physics; it sets out to provide a description of the present state of nuclear structure measurements and to demonstrate what can be learned by the application of quantum mechanics to nuclear models. A previous well founded knowledge of atomic physics, relativity and quantum theory and some acquaintance with the rudiments of nuclear physics are required for maximum benefit to be obtained from this book, as the containment of the material within the limits of a single volume is achieved by very compressed treatment of matters assumed covered in earlier courses. Space is thus gained for a thorough treatment of the single particle shell model, the collective model and the unified model. The final three chapters in which the whole field is reviewed from the standpoint of the electromagnetic, the weak and the strong interactions are particularly valuable. A generous supply of numerical problems is included chapter by chapter and the solutions of a selected number of these are given in an appendix.

The final-year undergraduates for whom the book is designed will in no sense find this an easy text. Those among them who have postgraduate research ambitions will find it an excellent base on which their future studies in nuclear or elementary particle physics can be securely built. In fact for several years to come the text should be suitable reading for postgraduate courses in the field. The volume is put together with the neatness and the authority to be expected from an experienced and respected practitioner in this area of research. 